International Journal of Modern Physics D

(C) World Scientific Publishing Company

\title{
CORRECT STATEMENT OF A SCATTERING PROBLEM FOR QUANTUM CHARGED SCALAR PARTICLES ON THE REISSNER-NORDSTRÖM BLACK HOLES *
}

\author{
N. E. FIRSOVA \\ Institute for Mechanical Engineering, Russian Academy of Sciences \\ Sankt-Petersburg 199178, Russia \\ Received : August 1997
}

\begin{abstract}
We study a correct statement of the scattering problem arising for quantum charged scalar particles on the Reissner-Nordström black holes when taking into account the own electric field of black hole. The elements of the corresponding $S$-matrix are explored in the form convenient to physical applications and for applying numerical methods. Some further possible issues are outlined.
\end{abstract}

\section{Introduction}

As is well known (see, e. g., Ref. 1 ), when calculating miscellaneous quantum effects in the $4 \mathrm{D}$ black hole physics one encounters many scattering problems. These problems can be split into two classes: the ones on the semiaxis and the ones on the axis. The former class is important when analysing, for example, the processes of the particle absorption by black holes. The latter one, to our mind, more important for the $4 \mathrm{D}$ black hole physics, is connected with semiclassical quantization of various physical fields on the background of the black holes and with quantum effects arising within the framework of this approach, for instance, such as the Hawking radiation process. Under the circumstances the situation with a more or less strict study of the mentioned problems should, however, be considered as unsatisfactory.

As a rule, the investigators treat the given problems at a qualitative level using one or another approximation without any justification from mathematical point of view. But many effects in the $4 \mathrm{D}$ black hole physics can be computed only numerically and when doing so it is very important to know whether the quantities under evaluation exist in the strict mathematical sense. The given quantities, however, often contain the characteristics related with the mentioned scattering problems, for example, the elements of the corresponding $S$-matrices. It is evident that in this situation the physical value of such computations is extremely low and they can serve only as a crude approximation.

The present note is intended to consider correctly one of the abovementioned scattering problems which arises when studying topologically inequivalent config-

*PACS Nos.: 03.65.Nk, 03.80.+r, 04.70.Dy 
urations (TICs) of complex scalar field on the 4D black holes. As was discussed in Refs. 2 , the standard spacetime topology $\mathbb{R}^{2} \times S^{2}$ of the $4 \mathrm{D}$ black hole physics admits the countable number of TICs for the complex scalar field, each TIC being labeled by its Chern number $n \in \mathbb{Z}$. In its turn, this yields, for instance, the additional contributions to the Hawking radiation due to the twisted TICs, i. e., the ones with $n \neq 0$. From physical point of view such contributions are conditioned by the natural presence of Dirac magnetic $U(1)$-monopoles on black holes. This question was explored in Refs. 3 . for the Scwarzschild(S) and Reissner-Nordström (RN) black holes. Referring for more details to Refs. expression for the luminosity $L(n, \alpha)$ with respect to the Hawking radiation for TIC with the Chern number $n$ for the case $\mathrm{S}$ and RN black holes. It looks as follows

$$
L(n, \alpha)=2 A \sum_{l=|n|}^{\infty}(2 l+1) \int_{0}^{\infty} B \frac{\left|s_{11}(k, \alpha, l)\right|^{2} k d k}{e^{4 \pi k[1+f(\alpha)]}-B}
$$

with dimensionless $k$ and $f(\alpha)=\frac{1}{2}\left(\sqrt{1-\alpha^{2}}+1 / \sqrt{1-\alpha^{2}}\right)$, while $A=\frac{1}{2 \pi \hbar}\left(\frac{\hbar c^{3}}{G M}\right)^{2} \approx$ $0.273673 \cdot 10^{50} \mathrm{erg} \cdot \mathrm{s}^{-1} \cdot M^{-2}(M$ in $\mathrm{g}), e=4.8 \cdot 10^{-10} \mathrm{~cm}^{3 / 2} \cdot \mathrm{g}^{1 / 2} \cdot \mathrm{s}^{-1}, \alpha=|Q| / M$ with $0 \leq \alpha<1, M, Q$ are, respectively, a black hole mass and an electric charge, and $B=\exp \left[-4 \pi e Q \frac{\sqrt{G}}{\hbar c} \frac{1+f(\alpha)}{1+\sqrt{1-\alpha^{2}}}\right]$.

In formula (1) we assume that $e Q>0$ or else it should be regularized because then there is a singularity in the integral.

To find the coefficient $s_{11}$, one should consider a scattering problem on the axis for the Schrödinger type equation

$$
\begin{gathered}
{\left[-\frac{d^{2}}{d x^{2}}+q(x, \alpha, l)\right] \psi(x, k, \alpha, l)=\left(k+\frac{e Q}{y(x)}\right)^{2} \psi(x, k, \alpha, l),} \\
q(x, \alpha, l)=\left[1-\frac{2}{y(x)}+\frac{\alpha^{2}}{y^{2}(x)}\right]\left[\frac{N}{y^{2}(x)}+\frac{2}{y^{3}(x)}-\frac{2 \alpha^{2}}{y^{4}(x)}\right],
\end{gathered}
$$

where $y(x)$ is a function reverse to the following one

$$
\begin{gathered}
x(y)=y+\frac{y_{+}^{2}}{y_{+}-y_{-}} \ln \left|\frac{y-y_{+}}{2}\right|-\frac{y_{-}^{2}}{y_{+}-y_{-}} \ln \left|\frac{y-y_{-}}{2}\right|, \\
y_{ \pm}=1 \pm \sqrt{1-\alpha^{2}},
\end{gathered}
$$

so $y(x)$ is the one-to-one correspondence between $(-\infty, \infty)$ and $\left(y_{+}, \infty\right)$. Besides, $N=l(l+1)$ or $N=l(l+1)-n^{2}$ (in both the cases $\left.l=|n|,|n|+1, \ldots\right)$ in dependence of the gauge choice for connection $A_{\mu}$ (vector-potential for the conforming Dirac monopole) in the line bundle with the Chern number $n$. Both the gauges are interesting from physical point of view but for the considerations in the present paper the concrete choice is inessential, therefore, in what follows, the notation $q(x, \alpha, l)$ can be taken as the common one for both the cases. 
In $4 D$ black holes physics literature (see, e. g., Ref. 1 ) it is proposed to determine the scattering functions as the solutions of the equation (2) which obey the following conditions

$$
\begin{gathered}
\Psi^{1}(x, k, \alpha, l) \sim \begin{cases}e^{i\left(k+e Q / y_{+}\right) x}+s_{12}(k, \alpha, l) e^{-i\left(k+e Q / y_{+}\right) x}, & x \rightarrow-\infty, \\
s_{11}(k, \alpha, l) e^{i k x}, & x \rightarrow+\infty,\end{cases} \\
\Psi^{2}(x, k, \alpha, l) \sim \begin{cases}s_{22}(k, \alpha, l) e^{-i\left(k+e Q / y_{+}\right) x}, & x \rightarrow-\infty, \\
e^{-i k x}+s_{21}(k, \alpha, l) e^{i k x}, & x \rightarrow+\infty\end{cases}
\end{gathered}
$$

with $S$-matrix

$$
S(k, \alpha, l)=\left(\begin{array}{ll}
s_{11}(k, \alpha, l) & s_{12}(k, \alpha, l) \\
s_{21}(k, \alpha, l) & s_{22}(k, \alpha, l)
\end{array}\right) .
$$

In the case of the $\mathrm{S}$ black hole, one should take $\alpha=Q=0$ and we come to the standard scattering problem on the axis for the Schrödinger equation which

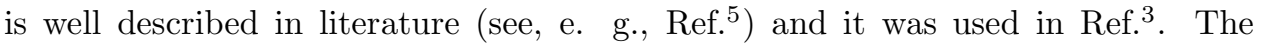
next important case is the RN black hole when neglecting the influence of its own (external) electric field on the charged scalar particles leaving the black hole. In this situation, one should take $e=0$ in (2), (6), (7), so that $B=1$ in (17), and we again come to the standard scattering problem for the Schrödinger equation but with the more complicated potentilal (3), where $\alpha \neq 0$. This case was analysed in Ref.t. It is clear that the most general case with taking into account the own electric field of RN black hole requires considering the scattering problem for (2) at arbitrary $e \neq 0, Q \neq 0, \alpha \neq 0$. This note is devoted just to this case. It is clear that there are difficulties in this problem since the potential $q(x, \alpha, l)$ of (3) is given in an inexplicit form. Besides, as will be seen below, the above statement of the scattering problem (see (2), (6)-(7)) is not quite correct at $e \neq 0, Q \neq 0, \alpha \neq 0$ and needs some changes. The reason is that the problem (2), (6) $-(7)$ has no solution since the effective potential of the problem is equal to

$$
q(x, \alpha, l)-2 k \frac{e Q}{y(x)}-\frac{e^{2} Q^{2}}{y^{2}(x)}
$$

and it is not integrable at $x \rightarrow+\infty$ (long range potential, see below), though the potential $q(x, \alpha, l)$ is integrable 6 . Therefore the problem should be regularized.

We start in Sec. 2 by obtaining the necessary estimates needed for the further correct statement of scattering problem under consideration. In turn, this allows us to formulate the mentioned problem in Sec. 3 while Sec. 4 contains the study of some asymptotic properties of the conforming $S$-matrix that can be useful, for example, for checking numerical calculations. Finally, Sec. 5 is devoted to concluding remarks.

Physical issues of the results obtained here will be discussed elsewhere, as they require numerical calculations like those in Refs. 3 . and the results of the present paper give the necessary basis for these computations. 
N. E. Firsova

\section{Preliminaries}

To study equation (2) we should first explore functions $y(x)$ and $q(x, \alpha, l)$. From (14), (5) it follows that if $y$ varies from $y_{+}=1+\sqrt{1-\alpha^{2}}$ to $+\infty$ then $x$ varies from $-\infty$ to $+\infty$. We see that

$$
x_{y \rightarrow y_{+}}=\frac{y_{+}^{2}}{2 \sqrt{1-\alpha^{2}}} \ln \left|y-y_{+}\right|+O(1) \rightarrow-\infty
$$

So we have

$$
y(x)_{x \rightarrow-\infty}=y_{+}+O\left(\exp \left(\frac{2 \sqrt{1-\alpha^{2}}}{y_{+}^{2}} x\right)\right) .
$$

Taking into consideration that $y_{+}^{2}-2 y_{+}+\alpha^{2}=0$ we get

$$
\left(1-\frac{2}{y(x)}+\frac{\alpha^{2}}{y^{2}(x)}\right)_{x \rightarrow-\infty}=O\left(\exp \left(\frac{2 \sqrt{1-\alpha^{2}}}{y_{+}^{2}} x\right)\right),
$$

and (see (3))

$$
q(x, \alpha, l)_{x \rightarrow-\infty}=O\left(\exp \left(\frac{2 \sqrt{1-\alpha^{2}}}{y_{+}^{2}} x\right)\right) .
$$

It is clear that

$$
y(x)_{x \rightarrow+\infty}=x\left(1+O\left(\frac{\ln x}{x}\right)\right) .
$$

From (3) and (11), (9) we see

$$
q(x, \alpha, l)_{x \rightarrow+\infty}=O\left(x^{-2}\right) .
$$

From (9), (10) it follows that as $x \rightarrow-\infty$ the equation (2) has the form

$$
\left[\frac{d^{2}}{d x^{2}}+\left(k+\frac{e Q}{y_{+}}\right)^{2}\right] \psi(x, k, \alpha, l)=q^{-}(x, k, \alpha, l) \psi(x, k, \alpha, l),
$$

where

$$
\begin{gathered}
q^{-}(x, k, \alpha, l)=u^{-}(x, k, \alpha, l)+2\left(k+\frac{e Q}{y_{+}}\right) v^{-}(x, k, \alpha, l), \\
u^{-}(x, k, \alpha, l)=q(x, k, \alpha, l)-v^{-}(x, k, \alpha, l)^{2} \\
v^{-}(x, k, \alpha, l)=e Q\left(\frac{1}{y_{+}}-\frac{1}{y(x)}\right)
\end{gathered}
$$

Here

$$
\begin{aligned}
& u^{-}(x, k, \alpha, l)_{x \rightarrow-\infty}=O\left(\exp \left(\frac{2 \sqrt{1-\alpha^{2}}}{y_{+}^{2}} x\right)\right), \\
& v^{-}(x, k, \alpha, l)_{x \rightarrow-\infty}=O\left(\exp \left(\frac{2 \sqrt{1-\alpha^{2}}}{y_{+}^{2}} x\right)\right),
\end{aligned}
$$

and consequently

$$
\int_{-\infty}^{a}\left|u^{-}(x, k, \alpha, l)\right| d x<C(a), \int_{-\infty}^{a}\left|v^{-}(x, k, \alpha, l)\right| d x<C(a) .
$$


From (11), (12) it follows that as $x \rightarrow+\infty$ the equation (2) has the form

$$
\left[\frac{d^{2}}{d x^{2}}+\left(k^{2}+2 k \frac{e Q}{x}\right)\right] \psi(x, k, \alpha, l)=q^{+}(x, k, \alpha, l) \psi(x, k, \alpha, l),
$$

where

$$
\begin{gathered}
q^{+}(x, k, \alpha, l)=u^{+}(x, k, \alpha, l)+2 k v^{+}(x, k, \alpha, l), \\
u^{+}(x, k, \alpha, l)=q(x, k, \alpha, l)-\frac{e^{2} Q^{2}}{y^{2}(x)}, \\
v^{+}(x, k, \alpha, l)=e Q\left(\frac{1}{x}-\frac{1}{y(x)}\right) .
\end{gathered}
$$

Hence, as $x \rightarrow+\infty$ (see (11), (12))

$$
u^{+}(x, k, \alpha, l)=O\left(x^{-2}\right), \quad v^{+}(x, k, \alpha, l)=O\left(x^{-2}\right) .
$$

As a result

$$
\int_{a}^{+\infty}\left|u^{+}(x, k, \alpha, l)\right| d x<c(a), \int_{a}^{+\infty}\left|v^{+}(x, k, \alpha, l)\right| d x<c(a),
$$

The homogenous equation for (13) is

$$
\left[\frac{d^{2}}{d x^{2}}+\left(k+\frac{e Q}{y_{+}}\right)^{2}\right] \psi_{0}^{-}(x, k, \alpha, l)=0,
$$

and its general solution has the form

$$
\psi_{0}^{-}(x, k, \alpha, l)=C_{1}^{-} e^{i\left(k+\frac{e Q}{y_{+}}\right) x}+C_{2}^{-} e^{-i\left(k+\frac{e Q}{y_{+}}\right) x} .
$$

Let us now consider the homogenous equation for (18)

$$
\left[\frac{d^{2}}{d x^{2}}+\left(k^{2}+2 k \frac{e Q}{x}\right)\right] \psi_{0}^{+}(x, k, \alpha, l)=0 .
$$

Transforming it we obtain for

$$
\left.u(z, k, \alpha, l))=\psi_{0}^{+}\left(\frac{z}{2 i k}, k, \alpha, l\right)\right)
$$

the Whittaker equation 6

$$
u_{z z}^{\prime \prime}+\left\{-\frac{1}{4}-\frac{i e Q}{z}\right\} u=0 .
$$

A couple of the Whittaker functions $W_{-i e Q, \frac{1}{2}}(z), W_{i e Q, \frac{1}{2}}(-z)$ forms a fundamental system of solutions for the equation (26) because they have asymptotic behavior as follows 6

$$
W_{-i e Q, \frac{1}{2}}(z)=e^{\frac{1}{2} z} z^{-i e Q}\left(1+O\left(\frac{1}{z}\right)\right),|\arg z|<\pi, z \rightarrow \infty,
$$


N. E. Firsova

$$
W_{i e Q, \frac{1}{2}}(-z)=e^{\frac{1}{2} z}(-z)^{i e Q}\left(1+O\left(\frac{1}{z}\right)\right),|\arg (-z)|<\pi, z \rightarrow \infty .
$$

Hence the general solution of (26) is

$$
u(z, k, \alpha, l)=C_{1}^{+} W_{-i e Q, \frac{1}{2}}(z)+C_{2}^{+} W_{i e Q, \frac{1}{2}}(-z) .
$$

It means that the general solution of (25) has the form

$$
\psi_{0}^{+}(x, k, \alpha, l)=C_{1}^{+} W_{i e Q, \frac{1}{2}}(-2 i k x)+C_{2}^{+} W_{-i e Q, \frac{1}{2}}(2 i k x)
$$

and

$$
\begin{gathered}
W_{i e Q, \frac{1}{2}}(-2 i k x)=e^{i k x}|2 k x|^{i e Q} e^{\frac{\pi e Q}{2}}\left(1+O\left(\frac{1}{|k x|}\right)\right), x \rightarrow \infty, \\
W_{-i e Q, \frac{1}{2}}(2 i k x)=e^{-i k x} \frac{1}{|2 k x|^{i e Q}} e^{\frac{\pi e Q}{2}}\left(1+O\left(\frac{1}{|k x|}\right)\right), x \rightarrow \infty .
\end{gathered}
$$

We introduce also functions

$$
w_{ \pm i e Q, \frac{1}{2}}( \pm z)=W_{ \pm i e Q, \frac{1}{2}}( \pm z) e^{-\frac{\pi e Q}{2}},
$$

which are more convenient. They form the fundamental system of solutions of the equation (25)as well. We may write the general solution of (25) as follows

$$
\psi_{0}^{+}(x, k, \alpha, l)=c_{1}^{+} w_{i e Q, \frac{1}{2}}(-2 i k x)+c_{2}^{+} w_{-i e Q, \frac{1}{2}}(2 i k x)
$$

and

$$
\begin{gathered}
w_{i e Q, \frac{1}{2}}(-2 i k x)=e^{i k x} e^{i e Q \ln |2 k x|}\left(1+O\left(|k x|^{-1}\right), x \rightarrow \infty,\right. \\
w_{-i e Q, \frac{1}{2}}(2 i k x)=e^{-i k x} e^{-i e Q \ln |2 k x|}\left(1+O\left(|k x|^{-1}\right)\right), x \rightarrow \infty .
\end{gathered}
$$

The functions $w_{-i e Q, \frac{1}{2}}(z), w_{i e Q, \frac{1}{2}}(-z)$ are complex conjugate, i.e.

$$
\overline{w_{i e Q, \frac{1}{2}}(-2 i k x)}=w_{-i e Q, \frac{1}{2}}(2 i k x)
$$

and their Wronskian is equal to

$$
\left[w_{-i e Q, \frac{1}{2}}(2 i k x), w_{i e Q, \frac{1}{2}}(-2 i k x)\right]=2 i k
$$

\section{The Scattering Problem}

We denote $\psi^{-}(x, k, \alpha, l)$ the Jost type solution of the equation (13), i.e.

$$
\begin{gathered}
{\left[\frac{d^{2}}{d x^{2}}+\left(k+\frac{e Q}{y_{+}}\right)^{2}\right] \psi^{-}(x, k, \alpha, l)=q^{-}(x, k, \alpha, l) \psi^{-}(x, k, \alpha, l)} \\
\psi^{-}(x, k, \alpha, l)=e^{-i\left(k+\frac{e Q}{y_{+}}\right) x}+o(1), x \rightarrow-\infty
\end{gathered}
$$


Varying constants in (23), (24) according to the Lagrange method, we obtain the integral equation equivalent to the problem (39), (40)

$$
\begin{gathered}
\psi^{-}(x, k, \alpha, l)=e^{-i\left(k+\frac{e Q}{y_{+}}\right) x}+ \\
\frac{1}{k+\frac{e Q}{y_{+}}} \int_{-\infty}^{x} \sin \left[\left(k+\frac{e Q}{y_{+}}\right)(x-t)\right] q^{-}(t, k, \alpha, l) \psi^{-}(t, k, \alpha, l) d t .
\end{gathered}
$$

The convergence of the series of approximations for this equations follows from (17) as usual (see, e. g., Ref.5).

Differentiating equation (41) we obtain expression for derivative

$$
\begin{array}{r}
\left(\psi^{-}\right)_{x}^{\prime}(x, k, \alpha, l)=-i\left(k+\frac{e Q}{y_{+}}\right) e^{-i\left(k+\frac{e Q}{y_{+}}\right) x}+ \\
\int_{-\infty}^{x} \cos \left[\left(k+\frac{e Q}{y_{+}}\right)(x-t)\right] q^{-}(t, k, \alpha, l) \psi^{-}(t, k, \alpha, l) d t
\end{array}
$$

A couple of functions $\psi^{-}(x, k, \alpha, l), \overline{\psi^{-}(x, k, \alpha, l)}$ is the fundamental system of solutions for the equation (39) and

$$
\left[\psi^{-}(x, k, \alpha, l), \overline{\psi^{-}(x, k, \alpha, l)}\right]=2 i\left(k+\frac{e Q}{y_{+}}\right) .
$$

We denote $\psi^{+}(x, k, \alpha, l)$ the Jost type solution of the equation (18), i.e.

$$
\begin{gathered}
{\left[\frac{d^{2}}{d x^{2}}+\left(k^{2}+2 k \frac{e Q}{x}\right)\right] \psi^{+}(x, k, \alpha, l)=q^{+}(x, k, \alpha, l) \psi^{+}(x, k, \alpha, l),} \\
\psi^{+}(x, k, \alpha, l)=w_{i e Q, \frac{1}{2}}(-2 i k x)+o(1), x \rightarrow+\infty .
\end{gathered}
$$

Varying constants in (25), (34) according to the Lagrange method, we obtain the integral equation equivalent to the problem (44), (45)

$$
\begin{gathered}
\psi^{+}(x, k, \alpha, l)=w_{i e Q, \frac{1}{2}}(-2 i k x)+\frac{1}{2 i k} \times \\
\int_{x}^{+\infty}\left[w_{-i e Q, \frac{1}{2}}(2 i k t) w_{i e Q, \frac{1}{2}}(-2 i k x)-w_{i e Q, \frac{1}{2}}(-2 i k t) w_{-i e Q, \frac{1}{2}}(2 i k x)\right] \times \\
\times q^{+}(x, k, \alpha, l) \psi^{+}(t, k, \alpha, l) d t .
\end{gathered}
$$

The existence of the solution of this equation follows from (22) as usual. Differentiating equation (46) we obtain expression for derivative

$$
\begin{gathered}
\left(\psi^{+}\right)_{x}^{\prime}(x, k, \alpha, l)=-2 i k w_{i e Q, \frac{1}{2}}^{\prime}(-2 i k x)-e^{-\pi e Q} \times \\
\int_{x}^{+\infty}\left[w_{-i e Q, \frac{1}{2}}(2 i k t) w_{i e Q, \frac{1}{2}}^{\prime}(-2 i k x)+w_{i e Q, \frac{1}{2}}(-2 i k t) w_{-i e Q, \frac{1}{2}}^{\prime}(2 i k x)\right] \times \\
q^{+}(t, k, \alpha, l) \psi^{+}(t, k, \alpha, l) d t .
\end{gathered}
$$


N. E. Firsova

The pair of functions $\psi^{+}(x, k, \alpha, l), \overline{\psi^{+}(x, k, \alpha, l)}$ is the fundamental system of solutions for the equation (44) and

$$
\left[\psi^{+}(x, k, \alpha, l), \overline{\psi^{+}(x, k, \alpha, l)}\right]=-2 i k .
$$

We write now decomposition of the solution $\psi^{+}(x, k, \alpha, l)$ to the fundamental system $\underline{\psi^{-}(x, k, \alpha, l)}, \overline{\psi^{-}(x, k, \alpha, l)}$, and the solution $\psi^{-}(x, k, \alpha, l)$ to the pair $\psi^{+}(x, k, \alpha, l)$, $\overline{\psi^{+}(x, k, \alpha, l)}$. We obtain

$$
\left\{\begin{array}{l}
\psi^{-}(x, k, \alpha, l)=c_{11}(k, l) \psi^{+}(x, k, \alpha, l)+c_{12}(k, l) \overline{\psi^{+}(x, k, \alpha, l)} \\
\psi^{+}(x, k, \alpha, l)=c_{21}(k, l) \overline{\psi^{-}(x, k, \alpha, l)}+c_{22}(k, l) \psi^{-}(x, k, \alpha, l)
\end{array}\right.
$$

We call the matrix $\mathbf{C}=\left\{c_{i j}\right\}$ the transition matrix for the equation (2). Let us explore its elements. From (43), (48), (49) it follows

$$
\begin{gathered}
c_{11}(k, \alpha, l)=\frac{1}{2 i k}\left[\overline{\psi^{+}(x, k, \alpha, l)}, \psi^{-}(x, k, \alpha, l)\right], \\
c_{12}(k, \alpha, l)=\frac{1}{2 i k}\left[\psi^{-}(x, k, \alpha, l), \psi^{+}(x, k, \alpha, l)\right], \\
c_{21}(k, \alpha, l)=\frac{1}{2 i\left(k+\frac{e Q}{y_{+}}\right)}\left[\psi^{-}(x, k, \alpha, l), \psi^{+}(x, k, \alpha, l)\right], \\
c_{22}(k, \alpha, l)=\frac{1}{2 i\left(k+\frac{e Q}{y_{+}}\right)}\left[\psi_{+}(x, k, \alpha, l), \overline{\psi^{-}(x, k, \alpha . l)}\right] .
\end{gathered}
$$

Let us investigate the properties of elements $c_{i j}$. For this purpose we put equations (49) one into another. Then we have

$$
\begin{aligned}
& c_{11}(k, \alpha, l) c_{22}(k, \alpha, l)+c_{12}(k, \alpha, l) \overline{c_{21}(k, \alpha, l)}=1, \\
& c_{22}(k, \alpha, l) c_{11}(k, \alpha, l)+c_{21}(k, \alpha, l) \overline{c_{12}(k, \alpha, l)}=1, \\
& c_{11}(k, \alpha, l) c_{21}(k, \alpha, l)+c_{12}(k, \alpha, l) c_{22}(k, \alpha, l)=0, \\
& c_{21}(k, \alpha, l) \overline{c_{11}(k, \alpha, l)}+c_{22}(k, \alpha, l) c_{12}(k, \alpha, l)=0 .
\end{aligned}
$$

It follows from equations $(50)-(53)$

$$
\begin{gathered}
\gamma(k, \alpha) c_{11}(k, \alpha, l)=-\overline{c_{22}(k, \alpha, l)}, \\
\gamma(k, \alpha) c_{12}(k, \alpha, l)=c_{21}(k, \alpha, l),
\end{gathered}
$$

where

$$
\gamma(k, \alpha)=\frac{k}{k+\frac{e Q}{y_{+}}} .
$$

From (54)-(59) we get also

$$
\gamma(k, \alpha)\left(\left|c_{12}(k, \alpha, l)\right|^{2}-\left|c_{11}(k, \alpha, l)\right|^{2}\right)=1,
$$




$$
\gamma^{-1}(k, \alpha)\left(\left|c_{21}(k, \alpha, l)\right|^{2}-\left|c_{22}(k, \alpha, l)\right|^{2}\right)=1 .
$$

These equations can be written in the form

$$
\begin{aligned}
& \frac{1}{\gamma(k, \alpha)} \frac{1}{\left|c_{12}(k, \alpha, l)\right|^{2}}+\left|\frac{c_{11}(k, \alpha, l)}{c_{12}(k, \alpha, l)}\right|^{2}=1, \\
& \gamma(k, \alpha) \frac{1}{\left|c_{21}(k, \alpha, l)\right|^{2}}+\left|\frac{c_{22}(k, \alpha, l)}{c_{21}(k, \alpha, l)}\right|^{2}=1 .
\end{aligned}
$$

We introduce now solutions $\Psi^{+}(x, k, \alpha, l), \Psi^{-}(x, k, \alpha, l)$ of the equation (2) satisfying the following conditions

$$
\begin{aligned}
& \Psi^{+}(x, k, \alpha, l)= \begin{cases}e^{\left.i\left(k+\frac{e Q}{y_{+}}\right) x\right)}+s_{12}(k, \alpha, l) e^{-i\left(k+\frac{e Q}{y_{+}}\right) x}+o(1), & x \rightarrow-\infty, \\
s_{11}(k, \alpha, l) w_{i e Q, \frac{1}{2}}(-2 i k x)+o(1), & x \rightarrow+\infty,\end{cases} \\
& \Psi^{-}(x, k, \alpha, l)= \\
& \quad= \begin{cases}s_{22}(k, \alpha, l) e^{-i\left(k+\frac{e Q}{y_{+}}\right) x}+o(1), & x \rightarrow-\infty, \\
w_{-i e Q, \frac{1}{2}}(2 i k x)+s_{21}(k, \alpha, l) w_{i e Q, \frac{1}{2}}(-2 i k x)+o(1), & x \rightarrow+\infty .\end{cases}
\end{aligned}
$$

As a result, from $(49)$ we obtain

$$
\left\{\begin{array}{l}
\Psi^{+}(x, k, \alpha, l)=\overline{\psi^{-}(x, k, \alpha, l)}+s_{12}(k, \alpha, l) \psi^{-}(x, k, \alpha, l)= \\
s_{11}(x, k, \alpha, l) \psi^{+}(x, k, \alpha, l) \\
\frac{\Psi^{-}(x, k, \alpha, l)}{\psi^{+}(x, k, \alpha, l)}+s_{22}(k, \alpha, l) \psi_{21}(k, \alpha, l) \psi^{+}(x, k, \alpha, l)=
\end{array}\right.
$$

In the similar way, from (49) we get

$$
\begin{array}{r}
\Psi^{-}(x, k, \alpha, l)=\frac{\psi^{-}(x, k, \alpha, l)}{c_{12}(k, \alpha, l)}, \\
s_{21}(k, \alpha, l)=\frac{c_{11}(k, \alpha, l)}{c_{12}(k, \alpha, l)}, \quad s_{22}(k, \alpha, l)=\frac{1}{c_{12}(k, \alpha, l)},
\end{array}
$$

and

$$
\begin{array}{r}
\Psi^{+}(x, k, \alpha, l)=\frac{\psi^{+}(x, k, \alpha, l)}{c_{21}(k, \alpha, l)}, \\
s_{12}(k, \alpha, l)=\frac{c_{22}(k, \alpha, l)}{c_{21}(k, \alpha, l)}, \quad s_{11}(k, \alpha, l)=\frac{1}{c_{21}(k, \alpha, l)} .
\end{array}
$$

For example for the transition coefficient $s_{11}(k, \alpha, l)$ which stands in the expression (11) for the luminosity $L(n, \alpha)$ we have formula

$$
s_{11}(k, \alpha, l)=2 i\left(k+\frac{e Q}{y_{+}}\right) /\left[\psi^{-}(x, k, \alpha, l), \psi^{+}(x, k, \alpha, l)\right] .
$$

According to (63), (64) we get the unitarity relations for matrix $S=\left\{s_{i j}\right\}$,

$$
\gamma^{-1}(k, \alpha)\left|s_{22}(k, \alpha, l)\right|^{2}+\left|s_{21}(k, \alpha, l)\right|^{2}=1,
$$




$$
\gamma(k, \alpha)\left|s_{11}(k, \alpha, l)\right|^{2}+\left|s_{12}(k, \alpha, l)\right|^{2}=1
$$

\section{Asymptotic properties of $S$-matrix}

To find out asymptotic behavior of the elements of $S$-matrix as $k \rightarrow \infty$ we should at first obtain the representation formulas for Jost type functions $\psi^{ \pm}(x, k, \alpha, l)$. They are as follows

$$
\begin{gathered}
\psi^{-}(x, k, \alpha, l)=F^{-}(x) e^{-i\left(k+\frac{e Q}{y_{+}}\right) x}+\int_{-\infty}^{x} A^{-}(x, t) \psi^{-}(t, k, \alpha, l) d t \\
F^{-}(x)=\exp \left[i \int_{-\infty}^{x} v^{-}(x) d x\right]
\end{gathered}
$$

and

$$
\begin{gathered}
\psi^{+}(x, k, \alpha, l)=F^{+}(x) w_{i e Q, \frac{1}{2}}(-2 i k x)+\int_{x}^{+\infty} A^{+}(x, t) \psi^{+}(t, k, \alpha, l) d t, \\
F^{+}(x)=\exp \left[i \int_{x}^{+\infty} v^{+}(x) d x\right] .
\end{gathered}
$$

We have here

$$
\left|\int_{x}^{ \pm \infty}\right| A^{ \pm}(x, t)|d t|<C\left|\int_{x}^{ \pm \infty}\left(\left|u^{ \pm}(x)\right|+\left|\left(v^{ \pm}\right)^{\prime}(x)\right|\right) d x\right| .
$$

To get formulas (73)-( $(76)$ one should replace $\psi^{ \pm}(x, k, \alpha, l)$ in the integral equation (41), (46) by its representations (73), (75) as usual (see Ref.6). Then we obtain the equations for $A^{ \pm}(x, t)$ and $F^{ \pm}(x)$. Solving equations for $F^{ \pm}(x)$ we get formulas (74), (76).

From (73)-(77) it follows that at $k \rightarrow \infty$

$$
\begin{gathered}
\psi^{-}(x, k, \alpha, l)=e^{-i k x} \exp \left\{i \int_{-\infty}^{1} v^{-}(x) d x-\frac{i e Q}{y_{+}}-i e Q \int_{1}^{x} \frac{d x}{y(x)}\right\}+o(1), \\
\psi^{+}(x, k, \alpha, l)=e^{i k x} \times \\
\exp \left\{i e Q \ln 2 k+i \int_{-\infty}^{1} v^{+}(x) d x-i e Q \int_{1}^{x} \frac{d x}{y(x)}\right\}+o(1) .
\end{gathered}
$$

It is not difficult to show that asymptotics (78), (79) can be differentiated. So, we have

$$
\begin{array}{r}
\left(\psi^{-}\right)^{\prime}(x, k, \alpha, l)=-i k e^{-i k x} \times \\
\exp \left\{i \int_{-\infty}^{1} v^{-}(x) d x-\frac{i e Q}{y_{+}}-i e Q \int_{1}^{x} \frac{d x}{y(x)}\right\}\left[1+O\left(\frac{1}{k}\right)\right],
\end{array}
$$




$$
\begin{array}{r}
\left(\psi^{+}\right)^{\prime}(x, k, \alpha, l)=e^{i k x} \times \\
\exp \left\{i e Q \ln 2 k+i \int_{-\infty}^{1} v^{+}(x) d x-i e Q \int_{1}^{x} \frac{d x}{y(x)}\right\}\left[1+O\left(\frac{1}{k}\right)\right] .
\end{array}
$$

From (78)-(81) it follows the asymptotic for Wronskian

$$
\begin{array}{r}
{\left[\psi^{-}(x, k, \alpha, l), \psi^{+}(x, k, \alpha, l)\right]=} \\
2 i k \exp \left\{i e Q\left[\ln 2 k-1 / y_{+}\right\} \exp (i V)\left[1+O\left(\frac{1}{k}\right)\right],\right.
\end{array}
$$

where

$$
V=\int_{-\infty}^{\infty} v(x) d x, v(x)=\left\{\begin{array}{ll}
v^{+}(x), & x \geq 1, \\
v^{-}(x), & x<1,
\end{array} .\right.
$$

Therefore from (52), (69) we have

$$
s_{11}(k, \alpha, l)=\exp \left\{-i e Q\left[\ln 2 k-1 / y_{+}\right]\right\} \exp (-i V)\left[1+O\left(\frac{1}{k}\right)\right] .
$$

In the same way we get asymptotics (see (51), (68)

$$
s_{22}(k, \alpha, l)=\exp \left\{-i e Q\left[\ln 2 k-1 / y_{+}\right]\right\} \exp (-i V)\left[1+O\left(\frac{1}{k}\right)\right]
$$

and (see also (50), (53))

$$
s_{12}(k, \alpha, l)=O\left(\frac{1}{k}\right), \quad s_{21}(k, \alpha, l)=O\left(\frac{1}{k}\right) .
$$

So, we have come to the conclusion that the correct statement of the scattering problem for the equation (2) at $e \neq 0, Q \neq 0, \alpha \neq 0$, should include the conditions (65)-(66) rather than the ones (6)-(7) accepted in the physical papers (see, e. g., Ref 1 and references cited therein). It may be understood from the physical point of view since the own (external) electric field of $\mathrm{RN}$ black hole is the Coylomb one. But as is known from the ordinary quantum mechanics (see, e. g., Ref.8) the Coulomb potential always requires special treatment so long as it is the long-range one.

\section{Concluding remarks}

We considered one of the scattering problems which encounter in the 4D black hole physics. Other ones will emerge when both the type of field and the type of black hole vary 1 . In principle, each field type (scalar, spinorial etc.) poses its own scattering problem which also depends on the type of black hole. It should also be taken into account that there can exist TICs for many fields on black holes and, as a consequence, this can increase the number of possibilities. But, as was mentioned early in the paper, the elements of the corresponding $S$-matrices are the important ingredients when calculating miscellaneous quantum effects connected with black holes. We hope, therefore, to continue the strict study of a number of the mentioned problems within the framework of our further investigations. 


\section{Acknowledgement}

The author is deeply thankful to Yu. Goncharov for drawing her attention to these

questions and for useful discussions. The work was supported in part by the Russian Foundation for Basic Research (grant No. 98-02-18244-a).

\section{References}

1. I. D. Novikov and V. P. Frolov, Black Hole Physics (Nauka, 1986). English edition: Physics of Black Holes (Kluwer Academic Publishers, 1989).

2. Yu. P. Goncharov and J. V. Yarevskaya, Mod. Phys. Lett. A9, 3175 (1994); A10, 1813 (1995);

Yu. P. Goncharov, Nucl. Phys. B460, 167 (1996).

3. Yu. P. Goncharov and N. E. Firsova, Int. J. Mod. Phys., D5, 419 (1996).

4. Yu. P. Goncharov and N. E. Firsova, Nucl. Phys., B486, 371 (1997).

5. K. Chadan and P. C. Sabatier, Inverse Problems in Quantum Scattering Theory (Springer-Verlag, 1977).

6. E. T. Whittaker and G. N. Watson, A Course of Modern Analysis (Cambridge University Press, 1973).

7. M. Jaulent and C. Jean, Ann. Inst. Henri Poincare, A25, 105 (1976).

8. L. L. Landau and E. M. Lifshits, Quantum Mechanics. Nonrelativistic Theory (Fizmatgiz, 1963);

A. I. Baz, Ya. B. Zeldovich and A. M. Perelomov, Scattering, Reactions and Decays in Nonrelativistic Quantum Mechanics (Nauka, 1971).

9. Yu. P. Goncharov, Phys. Lett. B398, 32 (1997). 